\title{
Vingt ans de relations entre le mouvement sportif et l'Etat : d'une collaboration ambivalente vers une régulation managériale?
}

\author{
Emmanuel Bayle, Christophe Durand
}

\section{Résumé}

Doté d'un poids social et économique $(1,7 \%$ du PIB) croissant, le sport constitue un secteur stratégique par les fonctions transversales et essentielles qui lui sont assignées par la société et les pouvoirs publics (éducation, santé, emploi, image de la France...). Le système sportif français repose, depuis plus de quarante ans, sur une "co-gestion" entre le mouvement sportif, représenté par les fédérations sportives nationales (organismes privés chargés d'organiser, de promouvoir et de développer leurs disciplines sportives et délégataires depuis 1984 d'une mission de service public) et l'Etat. Ce dernier, par l'intermédiaire du ministère des sports soutient la réalisation de cette mission et en contrôle l'exécution. Au cours des quatre dernières décennies, trois étapes sont distinguées dans les principes de régulation des rapports sport et Etat : une régulation autoritaire par l'affirmation du service public du sport (1960-1984), puis une crise de légitimité du modèle français de co-gestion du sport (1984-1996) et enfin une tentative de régulation managériale fondée sur un partenariat à géométrie variable (1996-2003). Pour expliquer ces étapes, nous montrons que le système de co-gestion du sport français est bâti sur des ambiguïtés et des paradoxes originels qui expliquent les stratégies d'acteurs qui ont vu le jour et notamment la stratégie d'accession à l'autonomie menée, au cours de ces dix dernières années, par la plupart des fédérations sportives nationales. Le constat d'échec de la relation d'agence qui en a résulté entraîne trois évolutions en cours : une tentative de repositionnement du pouvoir fédéral vers le centre du système sportif, une transformation du rôle du ministère de tutelle et plus largement une reconfiguration du système de régulation du secteur sportif fondé sur une nécessaire gouvernance partagée entre acteurs privés et publics que ce soit au niveau national, européen et international.

\section{Citer ce document / Cite this document :}

Bayle Emmanuel, Durand Christophe. Vingt ans de relations entre le mouvement sportif et l'Etat : d'une collaboration ambivalente vers une régulation managériale ?. In: Politiques et management public, vol. 22, $n^{\circ} 2,2004$. " Une génération de réformes en management public : et après ? » Actes du treizième colloque international - Strasbourg, jeudi 24 et vendredi 25 novembre 2003 - Tome 1. pp. 113-134;

doi : 10.3406/pomap.2004.2843

http://www.persee.fr/doc/pomap_0758-1726_2004_num_22_2_2843

Document généré le 11/06/2016 


\title{
VINGT ANS DE RELATIONS ENTRE LE MOUVEMENT SPORTIF ET L'ETAT : D'UNE COLLABORATION AMBIVALENTE VERS UNE RÉGULATION MANAGÉRIALE?
}

\author{
Emmanuel BAYLE* \\ Christophe DURAND**
}

Résumé

\footnotetext{
* Université Lyon 1 (CRIS EA 647).

** Université de Rouen (CETAPS JE 2318).
} 
Introduction

36 millions de Français déclarent pratiquer une activité physique et sportive et 14,4 millions de licences sont délivrées en France par les 175000 associations sportives.

350000 emplois seraient induits par le développement du sport dont plus de 200000 (publics et privés) dans le seul secteur sport. Le sport génèrerait 24,6 milliards d'euros (dont plus de 10 milliards d'euros apportés par les financeurs publics, principalement les communes) de dépense sportive globale soit $1,7 \%$ du PIB (Andreff et Nys, 2001). Au regard de telles donnees, le sport est devenu un secteur économique non négligeable. Au-delà, le sport constitue un secteur stratégique par les fonctions transversales et essentielles qui lui sont aujourd'hui assignees par la socièté et les pouvoirs publics : éducation (par le sport et la vie associative...), santé, insertion sociale (notamment des publics en difficulté), tourisme, aménagement du territoire, identité et image internationale de la France, support de relations internationales, de coopération et d'aide au développement... C'est pourquoi la culture française de service public et l'importance de telles fonctions ont généré, depuis le début des années 1960, une intervention massive de l'Etat dans le sport.

Le système sportif français repose ainsi, depuis plus de quarante ans, sur une "co-gestion" du sport entre le mouvement sportif, représenté par les fédèrations sportives nationales (FSN) - organismes privés sous statut associatif chargés depuis plus d'une vingtaine d'années d'une mission de service public - et l'Etat. Ce dernier, par l'intermédiaire du ministère des sports (MS) ${ }^{1}$, soutient la réalisation de cette mission et en contrôle l'exécution. Pour ce faire, les fédérations sportives nationales bénéficient d'aides étatiques directes et indirectes importantes. Ainsi quatre vingt fédérations unisports se partagent 150 millions d'euros répartis pour moitié par les 1600 cadres techniques fonctionnaires d'Etat mis à leur disposition et pour l'autre moitie sous la forme de subventions directes de fonctionnement. Ce modèle français, de par un fort niveau d'intervention des pouvoirs publics dans l'organisation du sport, dénote "une spécificité par rapport, notamment, aux pays du Nord de l'Europe traditionnellement beaucoup moins interventionnistes"2.

Cependant, on constate que ce système de co-gestion a profondément été déstabilisé au cours des vingt dernières années notamment en raison de transformations profondes de l'environnement du secteur : commercialisation intensive du spectacle et de la pratique sportive (professionnalisme), changement de la culture sportive, forte croissance et complexité de la réglementation, décentralisation des politiques publiques, construction de l'Union européenne (...). Ces phénomènes ont généré de nouveaux enjeux. Le rôle et les modes d'intervention des principaux acteurs s'en sont trouvés durablement modifiés.

\footnotetext{
1 Dans cet article, il est fait référence à la terminologie "ministère des sports" (appellation entre 2002 et mars 2004) et non au ministère de la jeunesse et des sports et de la vie associative (nouvelle dénomination depuis avril 2004) ou au ministère de la jeunesse et des sports (dénomination entre 1980 et 2002).

2 La taille des administrations publiques en charge du sport varie en conséquence : le ministère des sports français qui compte près de 7000 fonctionnaires contre quelques dizaines d'agents au Royaume-I!ni où la "sport and recreation division" est rattachée au secrétariat d'Etat chargé de la culture, des médias et du sport (Miege 2001).
} 
A la lumière de ces nouveaux enjeux, la question majeure que nous abordons est d'analyser comment l'Etat, s'est donné au cours des vingt dernières années, les moyens de faire face à cette évolution, notamment par une redéfinition de son rôle dans le système sportif. Dans cet article, nous tenterons de montrer que la relation Etat-fédérations sportives doit être examinée à travers les stratégies d'accession à l'autonomie menées par les FSN. La dynamique des relations, les changements de configuration de contrôle et finalement le système de régulation qu'elle implique pour l'Etat et son ministère de tutelle (MS) sont au centre d'un débat dont les enjeux se retrouvent dans beaucoup de secteurs où la tutelle publique étatique a largement été contestée ces dernières années.

La méthodologie adoptée consiste à avoir adopté un système d'observation et de veille sur la relation sport et Etat. Ainsi, plusieurs recherches empiriques ont èté menées sur le sujet (Durand 1994, Bayle 1996. Bayle 1999) à travers des monographies, des entretiens avec les acteurs du secteur et un suivi régulier des publications et revues de presse.

Sont ainsi observés :

- le niveau juridique, notamment l'évolution des dispositifs législatifs et conventionnels au cours des vingt dernières années :

- les aspects économiques, par le recueil des données financières sur la collaboration de l'Etat avec les 100 fédérations sportives nationales ;

- la dimension managériale, à travers l'analyse de la professionnalisation et de l'évolution, principalement sur les quinze dernières années, de la stratégie, des structures organisationnelles et des modes de gestion des FSN (...) ;

- l'évolution des systèmes de pilotage, de suivi et de contrôle des FSN mis en place par le ministère de tutelle.

Dans une optique comparative, les travaux portant sur la thématique de la régulation dans d'autres secteurs d'activité que le sport ont également été étudiés. L'observation d'organisations dont les logiques de fonctionnement sont mixtes et hybrides - car devant concilier logique commerciale et logique de service public - et qui se trouvent sous la tutelle des pouvoirs publics comme par exemple les secteurs culturel, sanitaire et social éclairent également la réflexion. Avec B. Demil et B. Leca (2003), nous pensons que pour étudier le système de régulation régissant une activité, "il faut considérer l'évolution des rapports de force entre les acteurs du champ considéré et la constitution d'équilibres entre eux. Ce faisant la constitution de l'environnement n'est pas exogène aux organisations mais dépend de leurs actions et des relais qu'elles parviennent à mettre en place". C'est pourquoi l'approche que nous proposons se traduit par l'utilisation de travaux historiques sur la collaboration Etat/FSN afin de repérer des périodes homogènes en termes de régulation et de comprendre le passage d'une période à une autre. Le concept de régulation fait référence, dans cet article, à une double définition :

- "l'aptitude d'un pilote à assurer le fonctionnement correct d'un système complexe" (Orange, 1999) ; 
- "la manière de codifier des règles légitimes ou de les recomposer ; c'est-àdire l'élaboration interactive et négociée des politiques publiques" (Gaudin, 1999).

Dans un premier temps, les ètapes les plus marquantes de l'évolution des rapports entre la tutelle étatique et le sport seront développées. Trois grandes pèriodes seront ainsi identifiées : la période 1960-1984; puis 1984-1996 et enfin 1996 à 2003.

La seconde partie propose une tentative d'explication des raisons pour lesquelles les rapports entre l'Etat et les FSN ont ainsi évolué.

\section{Les étapes de la régulation des rapports mouvement sportif et Etat}

La régulation des rapports mouvement sportif et Etat s'effectue depuis plus d'un siècle au nom de plusieurs principes dont limportance et la nature se sont modifiées avec le temps. Le premier principe est la volonté d'améliorer la condition physique de la nation pour la préparation de la guerre (fin du $19^{\text {ème }}$ siècle). Ensuite, l'obtention de la paix sociale et la formation d'une jeunesse forte et patriotique a èté recherchèe (dèbut du $20^{\text {ème }}$ siècle). C'est ensuite le prestige national qui est devenu l'enjeu majeur (années 1960). Enfin, le sport est aujourd'hui envisagé par la sphère publique comme un élément d'équilibre essentiel pour la société (années 1980). Vecteur de santé, de retombées économiques, d'emplois, d'insertion sociale, de cohésion et d'identité nationale, le sport apparaît à cet égard de plus en plus comme un outil de développement transversal et fédérateur au niveau éducatif, social, économique et identitaire pour la nation.

Au cours des quatre dernières décennies, trois étapes peuvent être distinguées dans les principes de régulation des rapports sport et Etat: une régulation autoritaire par l'affirmation du service public du sport, puis l'émergence d'une crise de légitimité du modèle français de co-gestion du sport et enfin une tentative de régulation managériale fondée sur un partenariat à géométrie variable.

\section{Une régulation autoritaire par l'affirmation du service public du sport (1960-1984)}

La première intervention législative majeure concernant le sport remonte à la charte des sports de $1940^{1}$ - et se situe dans un contexte politique très particulier. L'Etat étend, par la suite, ses visées "annexionnistes", notamment

\footnotetext{
1 La loi du 20 decembre 1940 (dite "charte des sports") relative à lorganisation sportive donne au gouvernement de Vichy des pouvoirs illimités. Cette charte vise à renforcer le lien entre l'ecole et l'Etat à travers les associations sportives et les fédérations; elle "étatise" presque complètement le mouvement sportif associatif. Chaque fédération est en effet placée sous le contrôle du Comité national des sports et "administrée par un comité de direction composé d'un président, d'un ou plusieurs vice-présidents, d'un secrétaire général, d'un trésorier désignés par arrêté du secrétaire d'Etat à l'instruction publique, et de membres élus pour moitié par l'assemblée générale et choisis par moitié par le secrétaire d'Etat à l'instruction publique". Source: loi du 20 décembre 1940.
} 
sur le sport de haut-niveau, au début des années 1960. Dans un climat international de "guerre froide", la représentativité nationale lors des compétitions sportives d'envergure devient un enjeu symbolique fort pour les nations. Cette implication régalienne, par le biais d'un système planificateur et centralisateur, se traduit par l'adoption de mesures qui concrétisent la mise en place de la première politique publique du sport en France ${ }^{1}$.

Cette politique entraîne alors une première rationalisation du fonctionnement des fédérations sportives. L'ambition déclarée est d'améliorer les résultats sportifs français dans les grandes compétitions internationales. Cet objectif entraîne une forme de "nationalisation" des fédérations sportives pour cause d'intérêt général et démontre la volonté de l'Etat de jouer "un rôle de tuteur effectif des fédérations" (Lachaume, 1991). Ce mouvement trouve son apogée dans la loi générale sur le sport du 16 juillet 1984 relative à l'organisation et la promotion des activités physiques et sportives. Si le sport est clairement défini comme relevant de la compétence de l'Etat, le législateur prévoit la délégation de cette mission de service public aux fédérations qui sont historiquement en charge de l'organisation, de la promotion et du développement du sport. II convient de noter que les fédérations sportives françaises dépendent d'un mouvement international d'institutions d'origines privées, mis en place à la fin du $19^{\text {ème }}$ siècle et resté en marge de l'intervention étatique. Le mouvement olympique, qui chapeaute ces organisations, se définit ainsi comme une philosophie 2 et organise des compétitions entre athlètes et non entre pays (art. 5 de la charte olympique). Ce modèle français d'organisation du sport va subir des contingences et des évolutions majeures au cours des années 1980.

\section{La crise de légitimité du modèle français de co-gestion du sport (1984-1996)}

La légitimité du modèle de co-gestion du sport est mise en cause par plusieurs raisons qui témoignent d'une crise du système de régulation.

La première raison est la mise en place des lois de décentralisation (1982, 1983) qui affaiblit de manière durable le rôle central de l'Etat dans le contrôle, la mise en cuvre et le financement des politiques sportives. Le modèle de co-

1 Elle s'est traduite par la réglementation du fonctionnement des fédèrations sportives, l'adoption de lois programmes (1961) relatives à la construction d'installations sportives, le développement des aides aux fédérations, la création d'un corps de techniciens du sport (rémunérés par l'Etat et mis à disposition des fédérations), la création d'un Conseil national du sport (1960) devenu Haut comité des sports (1961) pour permettre une concertation entre gouvernement et responsables sportifs. Une refonte du sport scolaire et universitaire est adoptée dans l'optique de rapprocher les activités physiques de l'école et celles du club afin d'améliorer le recrutement du sport de compétition. C'est également au milieu des années 1960 que l'Etat va s'attacher à réglementer les différentes professions liées au sport. De méme, c'est sous la cinquième république que sera généralisé le financement des fédérations sportives par l'Etat (à partir de 1959).

2 "Le but de l'olympisme est de promouvoir le sport au service d'un développement harmonieux de l'homme dans l'objectif d'encourager l'établissement d'une société pacifique et soucieuse de la préservation de la dignité humaine en éduquant la jeunesse à travers la pratique sportive sans discrimination de quelque sorte et dans l'esprit olympique, ce qui exige une compréhension mutuelle avec un esprit d'amitié, de solidarité et de fair-play" (source: Charte olympique, principes fondamentaux). 
gestion du sport se fissure et pose la question de savoir s'il peut laisser place à un modèle de gestion plus partagé et éclaté.

La deuxième raison est l'intensification de la commercialisation du spectacle et de la pratique du sport, avec notamment l'inflation continue des droits de télévision pour une dizaine de disciplines sportives, et l'augmentation des ressources de sponsoring, de quelques fédérations sportives (du fait de l'organisation de compétitions lucratives : Roland Garros, Tournoi des VI nations...). Le développement d'un financement d'origine commerciale devenu majoritaire dans quelques fédérations explique l'évolution importante de leurs comptes de résultat entre 1989 et 2002 : $+212 \%$ pour la fédération de football - sans prendre en compte le football professionnel géré par une ligue professionnelle -, $+236 \%$ pour le tennis, $+762 \%$ pour le rugby - sans prendre en compte le rugby professionnel géré par une ligue professionnelle. Cet important développement commercial pose la question de la place et de la légitimité du financement public pour des organisations développant majoritairement des prestations de services et, pour certaines, devenant des entreprises de spectacle sportif.

La troisième explication se trouve dans le changement général de la culture sportive du grand public. L'émergence du culte du corps, de la recherche du bien être personnel et la forte croissance du nombre de sports et des formes de pratique orientées vers le loisir et la pratique non encadrée (en dehors des clubs) remettent en cause le modèle pyramidal compétitif historique des fédérations sportives. Légitimé par l'Etat pour des impératifs de résultats sportifs internationaux, le sport compétitif élitiste est remis en cause. Cette évolution interroge la capacité du modèle fédéral aidé par l'Etat à faire face à ces changements et à répondre aux nouvelles demandes exprimées par les pratiquants loisirs.

Enfin, l'européanisation, dont le symbole est l'arrêt Bosman de 1995, pose notamment le principe de la libre circulation des sportifs professionnels au sein de I'Union européenne (UE) et la mondialisation du phènomène sportif complète l'explication. Les stratégies mondiales des marques de sports et l'internationalisation des compétitions et spectacles sportifs, entraînent, ainsi, des problèmes nouveaux ou en forte croissance: dopage, violence, changement de nationalité, difficultè à construire un modèle économique du sport professionnel européen ... Si ces "dérives" du sport tiennent à son développement, le niveau national apparaît de moins en moins pertinent pour réguler un certain nombre de problèmes en l'absence dune politique européenne claire en la matière de la part de l'UE.

Ainsi, c'est au cours de cette période que le phénomène sportif se segmente de plus en plus : sport loisir, sport de haut-niveau, sport spectacle, sport professionnel... Les FSN voient leur légitimité à gérer leur discipline sportive dans son ensemble contestée. Elles éprouvent de grandes difficultés à maintenir une cohérence entre le sport professionnel et le sport de haut niveau. Les opérateurs commerciaux empiètent peu à peu sur des activités jusqu'ici réservées aux systèmes fédéraux (événementiel, loisir...). 
Ces évolutions remettent ainsi en question le modèle de co-gestion français. Cette période est marquée par trois éléments majeurs traduisant une crise de la régulation du modèle français.

Tout d'abord, une très forte complexification du système législatif et réglementaire du sport français. On observe ainsi, au cours de ces quinze dernières années, près d'une dizaine de modifications de la loi de base sur le sport du 16 juillet 19841. Cette inflation législative marque une certaine instabilité et la difficulté de l'Etat à accompagner ou/et encadrer les évolutions sociales et économiques du secteur sportif d'autant plus qu'un certain nombre de textes n'ont pas étè réellement appliqués ${ }^{2}$.

Ensuite, la confrontation des ordres juridiques national et européens avec les normes et les règles des institutions sportives fait naître des contradictions. Ces dernières s'observent notamment en matière d'organisation du sport professionnel (notamment sur la libre-circulation des travailleurs au sein de l'Union européenne et quant au monopole de commercialisation des compétitions sportives dont bénéficient les instances historiques du sport), de dopage (difficulté d'harmonisation de la loi française à la réglementation internationale fédérale) et de normes d'équipements sportifs. Elles ajoutent aux incertitudes et à la complexité du système réglementaire sportif.

Enfin, les systèmes de contrôle du ministère des sports sur les fédérations sont apparus peu partiels et contestables. Ils n'ont pas empêché des déficits financiers fortement médiatisés d'une dizaine de fédérations au milieu des années 1990. Que ce soit à travers la découverte de malversations financières, de situations économiques très précaires de certaines FSN, de conflits politiques internes médiatisés, de pratiques de management contestées par leur opacité, les conditions de gestion et l'efficacité de certaines fédérations ont parfois été fortement mises en cause au cours des années $1990^{3}$.

1 Loi du 7/12/1987 - modifications sur les structures juridiques du sport professionnel -, loi "Bredin" du 13/07/1992 - refonte globale de la loi sur le sport du 16/07/1984 -, loi "Alliot-Marie" du 6/12/1993 sur la sécurité des enceintes sportives : loi "Pasqua" du 21/01/1995 d'orientation sur la sécurité dans les enceintes sportives, la loi du 6/03/1998 sur le droit à l'information; loi "Ayrault" du 28/12/1999 portant sur les subventions et les statuts des clubs professionnels ; loi "Buffet" du 6/07/2000 apportant une refonte d'envergure ; la loi "Lamour" du 1/08/2003 modifiant les statuts-types des fédérations, les conditions de l'enseignement rémunéré et de financement du sport professionnel

2 L'actuel ministre des sports, J-F. Lamour, déclarait à ce sujet: "il faut éviter de légiférer et de réglementer sans s'inquiéter, comme ces dernières années, des conditions réelles d'application des textes", Le Monde du 18/09/2002

3 Les fédérations françaises de hockey sur gazon, de base-ball, de football américain, de squash, de karaté (placée sous surveillance et suspension de la délégation et des subventions en 1998), de ski (comparution du président de la FFS pour détournement de fonds), des sports de glace et la ligue promotionnelle de volley-ball (détournement de fonds) se sont trouvées dans des situations financières particulièrement difficiles au cours des annèes 1990 .

Le cas de la Fédération française des sports de glace (51 MF de déficit en 1997 provoqué par les 28 responsables autorisés à signer des chèques au sein des 7 comités) est particulièrement symptomatique. Placée en redressement judiciaire le 7/71997, sa liquidation fut évitée avant les JO de Nagano en 1998 grâce au soutien du MS. Son ancien président, évincé sous la pression ministérielle, s'est retrouvé par la suite prestataire de services de la fédération (pour un montant de 300000 euros de 2000 à 2001) pour négocier les 
Le ministère de tutelle n'agit que tardivement en cas de dérapage financier des fédérations, facilitant peu l'apprentissage managérial des FSN. Pourtant, les conventions d'objectifs ministère/fédération, créées en 1985, semblaient porteuses de progrès. Si elles ont permis d'afficher les objectifs attendus et de créer dans les fédérations une dynamique objectifs/résultats notamment sur l'organisation du haut-niveau, ces conventions d'objectifs ont malgré tout longtemps constitué plus un dispositif formel de collaboration quant à l'affectation des subventions qu'une réelle culture du contrôle voire de l'évaluation.

Ainsi, le MS n'a finalement pas utilisé cet outil pour adapter et modifier sa stratégie de financement envers les FSN; ces dernières se retrouvent globalement chaque année avec une enveloppe budgétaire constante et leurs débordements stratégiques et/ou financiers sont majoritairement régulés par les jeux et les pressions politiques. Cette analyse permet de constater l'échec de la relation d'agence au cours de cette période.

Au cours des années 1980-1990, les moyens ministériels deviennent, de plus, trop faibles pour mener une politique sportive étatique ambitieuse.

Ces analyses traduisent finalement une crise de la régulation des outils publics (loi, convention, système de contrôle) au profit d'une régulation ambivalente et souvent de nature politique

Dans le même temps, le phènomène sportif devient, au cours des annèes 1990, un "phénomène social total" et l'Etat, dans cette perspective, assigne de nouveaux objectifs plus ambitieux aux fédérations sportives : lutter contre le chômage, les exclusions sociales, favoriser le développement économique à travers le sport (industrie, tourisme, emplois directs et induits...), participer à l'aménagement du territoire... Cette gestion d'objectifs pluriels est parfois impossible à mener face aux difficultés structurelles et au développement stratégique et organisationnelle fortement différenciés des fédérations.

Finalement, l'écart se creuse entre le sport de compétition placé sous le contrôle de l'Etat et les sports loisirs largement investis par la sphère privée commerciale. De son côté, le sport professionnel se trouve écartelé entre collectivités locales et investisseurs privés. La capacité du modèle français de co-gestion à "gérer tout le sport" de manière légitime et efficace est finalement fortement mise en cause. Ce sont les modes d'intervention du ministère des sports à l'intérieur du modèle qui vont être modifiés exprimant une tentative de régulation managériale.

droits de TV de cette féderation alors que son ancien DTN, fonctionnaire du ministère des sports devenait président de cette fédération.

La fédération d'haltérophilie, placée en redressement judiciaire le 29 avril 1997, présentait un passif entre 2.5 et $5 \mathrm{MF}$. Pour la première fois l'agrément ministériel a été retiré à une fédération délégataire d'une mission de service public et une fédération a connu une liquidation judiciaire. 


\section{Une tentative de régulation managériale fondée sur un partenariat à géométrie variable (1996-2003)}

L'année 1996 des jeux olympiques d'Atlanta correspond à la première tentative gouvernementale, très symbolique, de toucher aux fondements du modèle français d'organisation du sport. Le ministère des sports, sous la pression du ministère des finances, annonce son désengagement par la suppression de la mise à disposition auprès des fédérations d'environ 1600 fonctionnaires d'Etat. Le dispositif prévoyait un subventionnement équivalent, permettant aux FSN d'éventuellement salarier ces cadres.

Ce projet, qui n'a finalement pas abouti, illustre un changement de doctrine politique et une volontè de "privatiser" le système sportif et au delà de réduire le nombre de fonctionnaires dans un certain nombre de secteurs. II marque une apparente recherche de transparence et de régulation dans le modèle de co-gestion du sport français 1

Ce contexte explique une recherche d'information quantitative et qualitative plus importante sur le fonctionnement des fédérations (constitution d'une base de données financières sur les fédérations; d'un contrôle plus strict de l'affectation des fonds publics ministériels et de l'utilisation de ces derniers par les fédérations). Les difficultés de gestion des fédérations sont désormais mieux appréhendées grâce à la mise en place d'un certain nombre de dispositifs notamment depuis le début des années 2000 : obligation de règlement financier, mise en place d'une cellule d'alerte, de suivi et de contrôle des fédérations et par le travail de l'inspection générale du ministère des sports.

La volontè d'une rationalisation dans l'attribution des aides publiques directes et indirectes apparaît simultanément. Le MS considère les besoins rèels de chaque fédération et leur potentiel d'autonomie de financement. II tente également de réduire les inégalités entre fédérations sportives à partir d'une stratégie de redéploiement des cadres techniques des fédérations "les mieux dotées" (football, ski, athlétisme notamment) vers les fédérations les "moins dotées"

L'amélioration des dispositifs de conventions d'objectifs est recherchée à la fin des annèes 1990. La mise en place d'une aide aux politiques de développement de la pratique et l'aide à la structuration et à la professionnalisation des fédérations sont privilégiées. Par exemple, le dispositif sport/emploi et le dispositif emploi-jeune ont été des très forts accélérateurs de cette stratégie. Le MS a favorisé la création de 20000 emplois jeunes dans le secteur du sport associatif au cours des années

\footnotetext{
1 If faut dire que le MS rencontrait le risque de se voir qualifier de dirigeant de fait de certaines fédérations (au regard du pouvoir détenu par certains cadres techniques d'Etat mis à disposition auprès de fédérations et de l'importance du financement public apporté) et voir son contrôle sur ces organisations fortement mis en cause.
} 
$1997 / 2002$ soit une augmentation d'au moins $20 \%$ des emplois dans le mouvement sportif 1 .

Outre l'aide à la professionnalisation des fèdèrations, le MS tente d'organiser et de réguler la solidarité symbolique entre le sport professionnel et de hautniveau. Ainsi, en 2000, est créée, une taxe sur les droits TV redistribuée au sport de masse via le fonds national de développement du sport ${ }^{2}$. De même, une convention financière devient obligatoire entre les fédérations et leurs ligues sportives professionnelles (qui fonctionnent sous la tutelle de leur fédération délégataire). A travers de telles mesures, le MS se positionne finalement comme le garant législatif du monopole fédéral par lequel il tente de conserver et d'encourager le lien organique et financier entre sport de haut-niveau, sport professionnel et sport de masse (au niveau national avec les féderations au niveau local entre clubs pros/association support). Cette stratégie s'effectue en tentant de renforcer le modèle redistributeur au sein du mouvement sportif. II est remarquable de noter que cet axe stratégique à la base du "modèle français" a été conservé quelle que soit la couleur politique du ministre.

Cet aperçu chronologique sur l'évolution des rapports MS/FSN nécessite une tentative d'explication de ces étapes et des formes de régulation qui y ont vu le jour.

\section{Tentative d'explication des ètapes et des formes de régulation}

A partir du constat de l'existence d'ambiguïtés originelles, nous aborderons, ensuite, à travers le jeu des acteurs, les raisons d'un changement récent dans les relations MS et fédérations sportives nationales avant de conclure par une tentative d'analyse prospective relative aux modes de régulation future entre ces deux partenaires.

\section{Un système de régulation fondé sur des ambiguïtés et des paradoxes originels}

Comme l'analyse A. Loret (1989), on peut penser que l'association des fédérations à un service public leur a conféré alors un "statut d'utilité publique". Mais, appréhender l'institution sportive à travers son lien juridique étroit avec l'Etat revient, pour la plupart des auteurs, à l'associer à la politique de l'Etat et surtout à l'inféoder aux objectifs de ce dernier. C'est pourquoi B. Ramanantsoa et B. Moingeon (1992) parviennent à la conclusion que les FSN seraient des "extensions bureaucratiques de l'Etat".

\footnotetext{
1 Source: stats-infos (septembre 2003 http://www.jeunesse-sports.gouv.fr/ministere/stat-info.asp). II y aurait selon le conseil social du mouvement sportif de 70 à 120000 emplois équivalents temps plein dans les associations sportives.

2 Le FNDS (compte extrabudgétaire du trésor) représente flus du tiers du budget du MS et augmente presque de moitié les moyens étatiques consacrés à la politique sportive.
} 
Au regard de ces analyses, les FSN semblent agir en qualité d'agent privé de politique publique. Elles constituent, ainsi, dans la typologie d'H. Mintzberg (1986) des coalitions externes dominées, où le seul acteur dominant apparaît l'Etat via l'intervention du MS ; c'est en tous les cas le postulat implicite des différents auteurs précités. Mais la question du degré de contrôle effectif de l'Etat sur les FSN est plus complexe, ambivalent et surtout très variable selon les situations des fédérations. De plus, les FSN ne sauraient être considérées comme des associations para-administratives, car, même si, encore aujourd'hui, pour certaines d'entre elles, leur financement provient majoritairement des fonds étatiques, elles ne fonctionnent pas "par et pour" les pouvoirs publics. Si l'Etat se désengageait de leur activité, leurs conditions de fonctionnement seraient certes sérieusement remises en cause mais les FSN continueraient de perdurer car le besoin qu'elles satisfont est profondément d'origine privé. C'est d'ailleurs pourquoi, il est rare que l'Etat se substitue aux FSN dans leurs choix stratégiques (c'est essentiellement le cas lors de crise financière ou politique particulièrement grave). II exerce en fait un contrôle ambivalent et souterrain sur ces organisations.

Malgre cette analyse, la situation de collaboration imposée et dans les faits apparemment "appréciée" reste ambivalente. De façon paradoxale, le poids de la tutelle étatique est parfois dénoncé par les élus fédéraux sous prétexte de protéger l'indépendance des fédérations et au-delà du mouvement sportif face à l'Etat. L'ambivalence est ici manifeste, elle traduit, comme le souligne A. Loret (1989), une "volonté de leurs dirigeants de prendre le pouvoir sans prendre le pouvoir". II y a là un paradoxe propre au monde associatif qui est proche ou "allié" des pouvoirs publics. P. Di Maggio et H.K. Anheier (1990) montrent que ces organisations sont présentées de manière contradictoire "à la fois comme protectrices du pluralisme et des préférences des minorités et comme gardiennes des privilèges des élites ; comme agents extragouvernementaux de la démocratie et comme agents de contrôle autocratique; comme source d'innovation et de paralysie; comme les instruments des régimes au pouvoir et comme leurs concurrents".

G. Simon $(1990 ; 1997)$ met en évidence, à la suite de ces différents travaux relatifs aux rapports MS/FSN, le même paradoxe. II montre ainsi que "les fédérations disposent d'une délégation de pouvoirs du ministre des sports qui est purement fictive puisque le ministre des sports n'a jamais eu le moindre pouvoir en matière sportive. Malgré cette fiction les fédérations disent : mon pouvoir d'organisation, mon monopole sur les compétitions, au sens d'ailleurs très large, je le tiens de l'Etat, elles ne disent pas j'ai le pouvoir de faire ça. Elles se reposent sur une base, une source de pouvoir qui est sanctionnée par la loi et qui donne un pouvoir réel (un pouvoir légitime) à l'activité sportive et qui conforte en effet le pouvoir fédéral" (1997). Toutefois, comme le souligne un dirigeant sportif, "la reconnaissance par l'Etat n'est pas un élément de la légitimité des fédérations. Toute la légitimité vient des valeurs sportives et olympiques. Sinon le sport associatif devient un produit parmi d'autres. La question est de savoir si les fédérations ont la capacité de répondre à cet enjeu"1.

1 Source : entretien avec un ancien président de fédération 
La nature de l'activité et le statut associatif s'opposant à l'exercice d'une véritable tutelle, la position du ministère des sports ne peut dès lors qu'être fortement ambivalente. De plus, s'il souhaite garder un contrôle politique efficace sur le secteur et les fédérations, le MS doit laisser les structures fédérales dans une certaine dépendance de son action. Malgré le développement de ressources propres de la plupart des fédérations au cours de cette période (entre 1989 et $2002,+140 \%$ de croissance en moyenne des comptes de résultats des fédérations), la dépendance de l'ensemble des fédérations olympiques $(38,5 \%$ en $1989,37 \%$ en 1997 et $33 \%$ en 2002$)$ et non olympiques ( $22 \%$ en moyenne en 2002 ) vis-à-vis du financement direct du MS, si elle a diminué, reste encore importante. De plus, si l'on étudie le taux global de dépendance (subvention et cadres techniques mis à disposition), la dépendance financière réelle pour les FSN olympiques dépasse le plus souvent les $50 \%$ du total du compte de résultat à l'exception du football et du tennis et des fédérations non olympiques disposant d'un fort développement commercial (rugby, pétanque et sports automobile notamment).

Au-delà de l'aspect financier, la stratégie de dèpendance s'opère également, sur les fédérations disposant d'un fort développement commercial, par des pressions politiques et législatives pour leur faire adopter certaines politiques notamment par l'intermédiaire des cadres techniques. La situation et l'influence des cadres d'Etat mis à disposition auprès des fédérations, surtout pour ceux investis de missions nationales (Directeur Technique National DTN-, Conseiller Technique National), seront variables selon le profil de la fédération et la mission qu'ils doivent réellement $y$ accomplir (direction technique ou également administrative et organisationnelle, voire direction générale...). Statutairement, leur position traduit une situation ambiguë (notamment pour le DTN) puisqu'ils sont placés sous la double autorité du ministre et du président de fédération 1 . Politiquement ensuite, leur rôle et leurs fonctions peuvent être également ambigus puisqu'ils pourront jouer un "rôle de tutelle permanent, indirect" (Lachaume, 1991) ; de plus, leur pouvoir "technique" leur confère parfois légitimité à jouer directement ou indirectement un rôle politique au sein de la fédération. A ce sujet, B. Ramanantsoa et $C$. Thièry-Baslé (1989) remarquaient, ce qui est toujours vrai aujourd'hui, que "le cheminement de cadre technique à un poste de dirigeant bénévole et notamment de président est chose courante dans le monde fédéral".

Ces ambiguïtés et ambivalences sont à l'origine de la crise de la régulation, de l'échec de la relation d'agence et des jeux d'acteurs qui se sont développés dans le secteur. Cette crise peut s'expliquer selon l'analyse de $R$. Laufer (1993) par la crise du critère administratif qui définissait la légitimité publique (qui est déjà latente, comme nous venons de le montrer, lors de l'affirmation d'un service public du sport) ${ }^{2}$.

1 Cf. sur la situation des cadres techniques mis à disposition le rapport critique de la Cour des comptes qui consacre, en 2003, un chapitre aux fédérations et au ministère des sports

2 Dès l'affirmation dans la loi sur le sport (art. 11 et 13 de la loi du 29 octobre 1975 et article 16 de la loi du 16/07/1984) de la délégation d'une mission de service public aux fédérations. les fédérations se voyaient accorder la prérogative "d'exercer leur activité en toute indépendance" et dans le méme temps elles étaient placees sous la tutelle du ministère de la jeunesse et des sports ! 
Crise de la régulation et jeux d'acteurs

Le panorama historique dressé illustre la régulation en action. Mais, cette dernière apparaît comme un processus dynamique et complexe. Elle dessine "un espace stratégique pour les acteurs du champ" comme l'ont montré B. Demil et B. Leca (2003) concernant le marché de l'exploitation cinématographique française.

L'évolution de ces dix dernières années marque la stratégie de recherche d'une plus grande autonomie menée par la plupart des fédérations vis à vis de leur tutelle dans le modèle de co-gestion du sport français. Cette stratégie a été facilitée par une professionnalisation 1 de leurs comportements de gestion mis en œuvre selon des rythmes, des intensités et des formes très différenciées. Le MS doit, cependant, faire face à des profils stratégiques, organisationnels et financiers des FSN très disparates. L'action du ministère au cours des années 1980, notamment par l'attribution de moyens via les conventions d'objectifs, a permis de donner un premier cadrage stratégique à certaines fédérations leur permettant de mieux remplir leur "métier de base" (organisation du haut niveau et de la pratique compétitive). Elle a également favorisé un relatif conservatisme et immobilisme pour les fédérations en raison des aides étatiques substantielles, récurrentes et stables et de la culture sportive et peu managériale des cadres fédéraux. Cette situation a été à l'origine du retard pris par certaines fédérations dans leur organisation administrative et financière et au-delà dans la réponse aux attentes de leurs parties prenantes (médias, pratiquants de loisirs...) et en matière de structuration managériale des fédérations.

Les principaux acteurs du changement et de la professionnalisation de leurs comportements de gestion ont été, en plus de leur ministère de tutelle, certaines entreprises partenaires (notamment des entreprises publiques telles qu'EDF avec les sports d'eau) qui en leur permettant de développer leurs fonds propres les ont obligè à prendre en compte des attentes nouvelles (aménagement du territoire, communication...).

Ces pressions ont ètè à l'origine de la mise en place de nouveaux systèmes de pilotage stratégique fondés sur une démarche plus globale et formalisée. Deux nouvelles stratégies ont finalement été développées par la plupart des fédérations pour développer leur autonomie vis à vis de leur ministère de tutelle, maintenir ou développer leur nombre de licenciès et plus globalement répondre aux attentes de leur environnement:

- la diversification s'exprimant par la création de nouvelles activités : sport loisir, sport à l'école, insertion sociale par le sport, sport professionnel (...);

1 La professionnalisation associative fait référence à un "processus où l'amateurisme cède le pas à la rationalisation, à l'efficience et à la conduite des actions par projet. Cette rationalisation ne se traduit pas forcément par la rémunération du personnel" (Chantelat, 2001). Elle peut également s'exprimer par le développement de la compétence des bénévoles élus (spécialisation des tâches, recrutement de "bénévoles à profil"...) 
- la recherche d'un rayonnement multidimensionnel en tentant de répondre par leurs activités à des problèmes de société : emploi, santé publique, développement durable, développement économique, éducation-insertion, tourisme (...).

Ces évolutions témoignent de l'élargissement du métier de quelques fédérations et des tentatives ambitieuses de positionnement de certaines d'entre elles vers la gestion d'intérêts collectifs autour et par le sport. Dans ce contexte, les rapports de dépendance avec l'Etat se sont modifiés au profit de certaines d'entre elles pour lesquelles le MS devient un partenaire certes important mais plus unique (autres ministères - Education nationale et Environnement notamment -, collectivités locales, entreprises privées sponsors -, télévisions...). Tout en développant leurs activités et en modifiant leur stratégie, elles font preuve d'une stratégie d'ambiguiité envers leur ministère de tutelle. Cette dernière se traduit par une faible transparence dans leur gestion financière vis-à-vis du MS. Elle s'opère souvent par la transmission à une filiale commerciale de tout ou partie des activités commerciales de la fédération (le MS a du mal à connaître la réalité des fonds gérés par ces structures). Cette stratégie s'opère également en maintenant une forte pression par lobbying politique individuel (via des pressions sur le ministre des sports et plus largement sur les hommes politiques) et collectif (via le Comite National Olympique et Sportif Français ${ }^{1}$ ), au regard du succès du système sportif français dans les grandes compétitions internationales depuis ces dix dernières années, afin de préserver les avantages publics acquis (financement directs et indirects).

On pourrait penser que des organisations fortement contraintes comme les FSN (par la forte dépendance financière de certaines d'entre elles vis à vis de l'Etat, par l'obligation d'adopter des dispositions statutaires obligatoires, de conventionner avec leur ministère de tutelle...) disposent d'une étroite liberté de manœuvre. Or ce sont les principaux acteurs du secteur qui par interaction et négociation ont construit et régulé l'espace dans lequel ils ont développé des stratégies complexes non seulement sportives mais également commerciales et politiques au regard de l'importance symbolique prise par le sport dans nos sociétés.

"Les acteurs interagissent et jouent ainsi sur les rapports de force constituant des alliances d'intérêt et de valeurs" (Demil et Leca, 2003). C'est pourquoi, le ministère de tutelle n'a pas toujours été en position de force et a dú négocier avec les autres acteurs publics et privés du secteur sportif pour garder sa légitimité.

Les jeux d'alliances à l'intérieur du mouvement sportif ont permis de développer cette stratégie de recherche d'autonomie vis-à-vis des pouvoirs publics et un positionnement en partenaire plutôt qu'en "vassal" (Alaphilippe, 1992) d'un certain nombre de fédérations.

La mutualisation de moyens, les jeux coopératifs développés au sein du mouvement sportif (même si la solidarité développée en son sein est encore

1 Institution représentant le comité international olympique sur le territoire national et regroupant les fédérations sportives nationales sur le plan national. 
faible) et entre les partenaires publics et privés ont généré l'existence d'un modèle du sport recomposé et plus éclaté. Le repositionnement des acteurs s'effectue en fait au regard des zones d'incertitude qu'ils maîtrisent: le MS grâce aux variables législative, financière et politique; et pour le mouvement sportif, par sa montée en puissance, sa résonance politique et sociale et par la prise de conscience collective des enjeux dont le sport est porteur pour la nation.

Vers de nouvelles formes de régulation et un nouveau rôle des acteurs

A la lumière des évolutions du secteur sportif et de son environnement, il semble qu'une tendance lourde se dessine. Aujourd'hui, il y a nécessité de mieux définir et clarifier le rôle des agents de la régulation du système sportif. Dans cette perspective, trois évolutions voient le jour :

- une tentative de repositionnement du pouvoir fédéral vers le centre du secteur sportif (nécessitant une capacité managériale accrue des FSN pour y parvenir),

- une transformation et une mutation du rôle du MS,

- la recherche d'une nécessaire gouvernance partagée (entre partenaires publics et privés nationaux et internationaux) pour réguler et protéger le "bien public mondial"1 qu'est devenu le sport.

\section{- Un repositionnement du pouvoir fédéral vers le centre du système sportif}

La priorité du MS et l'évolution de la stratégie de la plupart des FSN semblent avoir été de conforter et de favoriser le rôle central des fédérations dans le système sportif, de favoriser leur professionnalisation, l'assainissement de leurs modes de gestion et leur ouverture à leurs parties prenantes. La dernière évolution législative de la loi Lamour du 3 août 2002 permettant aux acteurs commerciaux d'entrer dans les comités directeurs fédéraux est une illustration concrète de cette évolution. Cette responsabilisation des FSN témoigne du passage, certes progressif et encore partiel, d'une logique bureaucratique de contrôle et d'une collaboration ambivalente vers une tentative de régulation plus managériale. Cette dernière s'exprime par une nécessaire clarification de la mission, des objectifs, de l'évaluation des FSN impliquant une obligation de "rendre compte" en termes de qualité, de quantité des prestations proposées et de coûts de leurs activités auprès de l'ensemble de leurs parties prenantes.

Pour ce faire, les fédérations doivent, cependant, démontrer leur capacité managériale. Bayle (1999) montre que cette dernière et le potentiel de

1 Cf. sur cette notion voir J.E. Stiglitz, (1999) et P. Hugon (2003). Ce concept signifie que le sport (au regard de ses valeurs et de ses externalités pour les sociétés, notamment en termes éducatifs et de santé publique) est devenu un bien collectif mondial ou global directement lié au contexte de la mondialisation. 
performance des FSN dépendent, plus précisèment, de leurs systèmes de gouvernance, de leurs modes de financements, de la qualité du maillage fédéral (qualité de la collaboration siège fédéral/ligues régionales/comités départementaux et clubs et de la capacité de ces derniers à mettre en œuvre les politiques fédérales 1 ) et de la stabilité et la qualité des dirigeants bénévoles et de leur personnel d'encadrement technique et managérial. Si la professionnalisation progresse (plus de professionnels qualifiés aux côtés des dirigeants bénévoles, des bénévoles parfois cooptés au regard de leurs compétences managériales), les systèmes managériaux fédéraux restent fragiles (cf. rapport Cour des comptes 2003) et reposent souvent sur quelques individus (le président et le DTN le plus souvent); ils connaissent actuellement des transformations culturelles majeures avec, depuis 2002, la rémunération de quelques présidents élus. Le rapport de la Cour des comptes 2003 constate notamment la faiblesse des outils de gestion dans les fédérations auditées: suivi des engagements insuffisants, comptabilité analytique sommaire et contrôle de gestion inexistant, absence d'identification des procédures d'engagement et de paiement, faiblesses des fonctions de contrôle interne et externe.

Selon la stratégie choisie explicitement ou implicitement, la fédération devra donc disposer des capacités en interne (moyens humains, matériels, financiers...) pour assumer les projets à mettre en œuvre. Dans tous les cas, cela semble impliquer une professionnalisation accrue des organisations (pensée stratégique), de la gestion et du contrôle des activitès et d'une très bonne collaboration entre les dirigeants élus et salariés. L'évolution de la stratégie des FSN implique également la mise en place de systèmes de cooperation et de mutualisation de moyens humains et matériels (avec d'autres fédérations, avec le CNOSF ou/et d'autres partenaires) pour assurer l'efficacité du développement des différents sports sur les aspects loisirs, tourisme, organisationnels... Les débuts d'organisation en famille de sports pour développer des concepts communs sur les pratiques de loisirs (notamment avec la création, dans les années 1990, du conseil supérieur du nautisme et du conseil des sports de pleine nature) marquent, à cet égard, une importante évolution. La stratégie de coopération avec le secteur commercial (industrie, distribution ou/et entreprises commerciales du secteur sportif) de certaines fédérations pour relancer le développement de leurs sports en atteste également.

A travers ces évolutions, la capacité managériale des FSN à mettre en œuvre et piloter des partenariats public/privé avec de multiples acteurs publics et privés est interrogée. Il leur faut inventer comme pour d'autres organisations ${ }^{2}$ un mode de gestion hybride qui combine finalement trois logiques de fonctionnement (associative, service public et commerciale) antinomiques mais paradoxalement complémentaires pour améliorer leurs performances et préserver leur légitimité (Bayle, 1999). C'est la combinaison de ces logiques

\footnotetext{
1 A cet égard, pour conserver leur base de licenciés et les fidéliser dans le système fédéral, les fédérations s'efforcent de développer de nouvelles formes de pratiques et d'inscrire leurs clubs dans une démarche qualité à travers une pratique de labellisation fondée sur différents critères: qualité des installations, niveau de formation des entraîneurs ... Ces formules de labellisation ressemblent à des systèmes de franchise (cf. Pigeassou et Chaze, 1999).
}

2 Cf. sur les musées les travaux de Bayard et Benghozi, 1993 - et de Sauiquin, 1996 - sur les cliniques. 
de fonctionnement qui doit leur permettre de développer un modèle de recherche du profit original, redistributeur, pour faire vivre des activités sociales déficitaires au cœur de leur projet (formation des sportifs, suivi social, aide à la structuration des clubs...). L'opposition de ces trois logiques de fonctionnement et la nécessité de les articuler rendent l'identité organisationnelle de ces organisations potentiellement conflictuelle (tensions dues à l'hétérogénéité et l'ambiguïté des objectifs). C'est pourquoi, leur stratégie consiste à produire de l'ambiguité vis à vis de leurs partenaires et à concilier selon une logique de compromis leurs obligations de service public, les exigences économiques et la culture associative à l'origine de leur légitimité

\section{- La transformation et la mutation du rôle du ministère des sports}

Récemment critiqué par le rapport de la Cour des comptes 2003 ("il ne semble pas que le MJS ait une vision claire des objectifs à atteindre, ni de la façon d'y parvenir"), le rôle du ministère des sports semble avoir pourtant évolué.

Face à l'arrivée de nouveaux acteurs et à la stratégie d'autonomie menée par certaines fédérations, le MS se positionne de plus en plus en observateur national de référence (sur l'emploi, la pratique sportive, les flux financiers du sport), en médiateur, organisant la concertation nationale 1 et internationale (sur le dopage, le sport professionnel, coopération internationale et francophonie, organisation des grands événements notamment ${ }^{2}$ ), en incitateur (à la professionnalisation des fédérations, la mise en place de partenariat), en contrôleur (des financements ministériels et de leur attribution). Traditionnellement centré sur des compétences juridiques et administratives (corps des inspecteurs de la jeunesse et des sports et quelques administrateurs civils) et sportive (corps des professeurs de sport) insuffisantes pour accompagner son évolution, le MS se dote progressivement de nouvelles compétences dans le cadre du recrutement de ces agents (statisticiens, spécialistes juridiques, gestionnaires, financiers...) visant à "renforcer les capacités de pilotage de l'administration centrale" 3 .

La partition du ministère de la jeunesse et des sports en 2002 , et le rattachement du secteur de la jeunesse et de l'éducation populaire au ministère de l'éducation nationale et de la recherche, a pu faire croire à un possible affaiblissement de ce ministère. Le nouveau rattachement de la jeunesse et de la vie associative au ministère des sports a renforcé la légitimité de cette institution. Finalement, le MS se positionne, de plus en plus comme le garant de l'unicité de l'organisation française (voire européenne) du

1 On peut noter la multiplication des organes de consultation et de cogestion du sport dont la plupart ont été créés à la fin des années 1990 : le conseil national des activités physiques et sportives (CNAPS), le conseil national de la recherche et de la technologie des APS, conseil national du FNDS, conseil national du sport de haut-niveau, conseil de prévention et de lutte contre le dopage, commission nationale des enceintes sportives. On remarquera également la création d'instances de débat et d'échanges avec les acteurs du monde sportif notamment dans une optique législative (les Etats gènéraux du sport en 2002).

215 championnats du monde ont été organisés en France en 2003.

3 Source : Réponse du ministère des sports au rapport de la Cour des comptes 2003 
sport en prenant de nombreuses mesures pour assurer à l'édifice sa pérennité dans le souci du respect du modèle pyramidal des compétitions (régulation du lien organique et financier sport de masse/ de haut niveau et professionnel) et du rôle social du sport. Ce positionnement nécessite la promotion d'une gouvernance partagée du sport que ce soit au niveau national ou international. Cette perspective rencontre encore cependant bien des difficultés notamment au niveau national. Comme le souligne le rapport de la Cour des comptes 2003, "l'articulation de l'action des collectivités territoriales avec l'intervention de l'Etat n'est pas organisée et la coordination des actions menées par les services déconcentrés du ministère des sports avec les instances chargées de mettre en œuvre la politique sportive des collectivités territoriales dépend des circonstances locales".

\section{Vers une nécessaire gouvermance partagée}

II semble que les principaux acteurs nationaux et internationaux, et notamment le MS, aient pris conscience que la régulation d'un bien public mondial comme le sport exige la mise en œuvre d'une gouvernance partagée. A cet égard, les initiatives, sous l'impulsion de l'Etat français, s'intensifient pour faire reconnaître, comme pour la culture, la spécificité du sport au niveau européen 1 .

Comme dans d'autres secteurs d'activité, l'impuissance et l'incapacité des Etats et du mouvement sportif à réguler seuls un certain nombre de dérives (violence, dopage, difficultés à construire un modèle du sport professionnel européen...) leur imposent de trouver une concertation et une régulation multiniveaux, infranationale et supranationale, pour gérer ces phénomènes. Cela entraîne une remise en cause de leurs modalités traditionnelles de gouvernement avec une recherche de concertation, de collaboration à travers la mise en cuvre de partenariats public/privé afin de coordonner et d'harmoniser les politiques nationales et d'améliorer leur efficacité. Le meilleur exemple en est l'évolution de la lutte contre le dopage : "il est intéressant de noter combien l'influence des Etats de l'UE a été déterminante dans la conception actuelle de l'agence mondiale anti-dopage (AMA). Les exigences de l'UE ont permis que le conseil de fondation de l'agence mondiale antidopage soit composé à parité de représentants du monde sportif et des gouvernements (l'Europe réclame une évolution des statuts vers une fondation de droit public afin de mettre en œuvre une harmonisation mondiale des contrôles et des procédures et de suppléer au manque de moyens de certains Etats dans la lutte contre le dopage" (Miège, 2001). Tout comme le dopage qui est perçu par de nombreux gouvernements comme un problème de santé publique (puisqu'il ne touche pas que les sportifs de haut-niveau mais aussi de nombreux autres sportifs), la violence et la corruption pourraient être de plus en plus vues comme des problèmes touchant à l'ordre,

$1 \mathrm{Cf}$. la déclaration sur le sport (sans valeur juridique) lors de la réunion des chefs d'Etat et de gouvernements de l'UE adoptée à Nice en décembre 2000 : "la communauté doit tenir compte, même si elle ne dispose pas de compétences directes, dans ce domaine, dans son action au titre des différentes dispositions du traité, des fonctions sociales, educatives et culturelles du sport qui fondent sa spécificité, afin de respecter et de promouvoir l'éthique et les solidarités nécessaires à la préservation de son rôle social". 
voire à la morale, publics. Chappelet (2002) montre qu'une agence indépendante comme l'AMA pourrait devenir une sorte "d'organisme de régulation du sport mondial".

Comme dans d'autres secteurs d'activités, cette nécessaire régulation supranationale (cf. Hugon 2003) entraîne une révision du rôle de l'Etat et la mise en place d'une nouvelle forme de régulation étatique : moins centrée sur la qualité d'opérateur de politiques publiques que sur un rôle de veille, de négociation. de médiation, de co-réglementation et de contrôle afin de tirer partie pour la nation et les pouvoirs politiques des externalités positives du sport et les valoriser (organisation d'événements internationaux majeurs, insertion sociale par le sport, développement de l'emploi sportif, image de "la France qui gagne"...). Ce repositionnement du rôle de l'Etat et de son ministère de tutelle marque une tentative de régulation managériale et s'exprime finalement par la volonté sous-jacente de "donner mieux pour valoriser et impulser plus" qui s'inspire des théories du "new public management".

Les derniers états généraux du sport (2002) et le projet de fondation du sport portés par le ministère des sports témoignent de cette volonté étatique de généraliser les stratégies "non-budgétaires"1 qui consiste à utiliser l'argent des autres apporté volontairement ou, ce qui revient au même, leur temps. La généralisation des stratégies non-budgétaires s'appuyant sur les associations et les fondations devrait, à l'avenir, réduire les prélèvements obligatoires, diminuer le coût de production des services, participer aux politiques de réduction du chômage et de réduction de la taille des administrations publiques.

Conclusion

Le développement économique du sport expose le modèle de co-gestion du sport français à une multiplicité de désordres potentiels qui ont entraîné une crise de la régulation du système sportif national au cours des années $1980 / 1990$. Elle s'est traduite par l'échec de la relation d'agence ministère des sports/fédérations sportives nationales prenant la forme d'une régulation très politique du secteur, permettant aux fédérations de développer une stratégie de recherche d'une plus grande autonomie. Nos analyses ne sont pas spécifiques au secteur étudié mais se retrouvent dans un certain nombre de secteurs (culturel, action sanitaire et sociale, éducation...). La dynamique de la relation étudiée et les changements de configuration de contrôle qu'elle implique notamment pour le ministère de tutelle montrent que l'on passe progressivement, depuis la fin des années 1990, d'une collaboration fortement ambivalente à une tentative de régulation managériale du système. Cette dernière s'exprime à la fois par la modification du périmètre et les modalités d'intervention que le MS s'efforce d'adopter et par l'introduction, certes parfois lente et souvent très inégale, d'une logique managériale au sein des fédérations et dans le système de pilotage du sport français. Dans un tel contexte, l'introduction de la dimension managériale et de ses principes constitue à la fois pour les FSN et pour son ministère de tutelle une nouvelle

1 Terme proposé par G. Orange, Peut-on parler d'un nouveau management ?. Site internet Economie-gestion www.educnet ed (conférence mars 2002). 
marque de modernité et de régulation comme le fut, dans les années 1960 , l'émergence des politiques publiques sportives. 


\section{BIBLIOGRAPHIE}

ALAPHILIPPE F., "Le pouvoir fédéral", Pouvoirs, n61, Paris, PUF, 1992, pp. 71-84.

ANHEIER H.K. et DI MAGGIO P., "The sociology of non profit organizations", Annual Review of Sociology, 16, 1990, pp. 35-46.

ARNAUD P., "Genèse des politiques sportives publiques", Revue française d'administration publique, $n^{\circ} 97$, janvier-mars 2001, pp. 29-38.

BAYART D. et BENGHOZI P.J., Le tournant commercial des musées en France et à l'étranger, Paris, ministère de la Culture et de la Francophonie, direction de l'administration gènérale et de la prospective, 1993.

BAYLE E., Management et performance des organisations à but non lucratif. Le cas des fédérations sportives nationales, thèse de doctorat de sciences de gestion, université de Limoges, 1999.

BAYLE E., "Mouvement sportif et Etat : la fin justifie-t-elle les moyens ?", Numéro spécial de la RJES relatif à l'avant-projet de loi sur le sport, décembre 1996, Editions Lamy, p. 81 à 92.

BAYLE E., DURAND C., "Sport professionnel et représentation nationale : quel avenir ?", Revue Reflets et perspectives de la vie économique : Numéro spécial Sport et mondialisation : Quel enjeu pour le XXlème siècle? Dir. J-J. Gouguet, Ed. De Boeck université, juin 2000, pp. 45-59.

CHANTELAT P. (Dir.), La professionnalisation des organisations sportives, Editions L'Harmattan, 2001.

CHAPPELET J.-L., "L'agence mondiale antidopage : un nouveau régulateur des relations internationales sportives", Relations internationales, $n^{\circ} 111$, automne 2002, pp. 381-401.

DEMIL $B$. et LECA $B$., "Architecture de marché et régulation dans l'exploitation cinématographique française", Revue française de gestion, Dossier spécial Manager les activités culturelles, juin $2003 n^{\circ} X X$ p. 229-252.

DURAND C., Essai d'analyse structurelle et de diagnostic des clubs sportifs professionnels, thèse de doctorat de gestion, IAE de Caen, 1994.

GAUDIN J-P., Vers un Etat régulateur?, Sciences humaines hors-série $n^{\circ} 44$, mars-avril-mai 2004.

GAUTRAT P., "Le sport dans les relations internationales", Revue française d'administration publique, $\mathrm{n}^{\circ} 97$, janvier-mars 2001, pp. 141-157.

GABORIT P., "Le ministère de la jeunesse et des sports", Revue Pouvoirs, n61, 1992, pp. 57-70.

GUYON C., "De la managérialisation du secteur public à un véritable métissage public-privé ?", Revue française de gestion, n¹15 septembre/octobre 1997, pp. 141-154.

HUGON P., "Les biens publics mondiaux: un renouveau théorique pour penser l'action publique à l'échelle mondiale", Revue Politiques et management public, vol. 21, septembre $2003 n^{\circ} 3$, pp. 55-72.

LACHAUME J.-F., "Du contrôle de l'Etat sur les fédérations sportives", Revue juridique et économique du sport, $n^{\circ} 16,1991$, pp. $3-20$.

LAUFER R., L'entreprise face aux risques majeurs, Logiques sociales, L'Harmattan, 1993.

LAUFER R., "Quand diriger c'est légitimer", Revue française de gestion, novembre-décembre 1996, pp. 51-62. 
MIEGE C., "Les organisations sportives et l'Union européenne: entre dérégulation et recherche de nouvelles règles", Revue française d'administration publique, $n^{\circ} 97$, janvier-mars 2001, pp. 5-14.

MINTZBERG $H$., Le pouvoir dans les organisations, Paris, Les Editions d'Organisation, 1986.

LORET A., L'utilité publique des organisations sportives face à l'évolution du sport contemporain. Du temps mesuré à la durée vécue, thèse de doctorat de sciences des organisations, université Paris Dauphine, 1989.

LORINO P., "A la recherche de la valeur perdue : construire les processus crèateurs de valeur dans le secteur public", Revue Politiques et management public, vol. $17, n^{\circ} 2$, juin 1999, pp. 21 à 33.

OLIVER C., "Determinants of interorganizational relationships : intégration and future directions", Academy of management review, 15/2/1990, pp. 241-265.

ORANGE G., "Peut-on parler d'un nouveau management ?", Site internet Economie-gestion (www.educnet.ed Conférence mars 2002).

ORANGE G., "Régulation", Encyclopédie de la gestion et du management, dir. R. LE DUFF, Dalloz, 1999.

PIGEASSOU C. et CHAZE J-P., "Paradoxes et enjeux des labels dans les services sportifs : outil politique de stratégie fédérale ou vecteur dynamique de développement économique local", in actes du colloque Le marketing du loisir et du tourisme sportif, dir. P. BOUCHET, 1999, p. 32-51.

RAMANANSTSOA B., THIERY-BASLE C., Organisations et fédérations sportives, Paris, PUF, Pratiques corporelles, 1989.

SAULQUIN J.-Y., La clinique en observation : formulation d'un diagnostic et relation à l'efficacité, thèse de doctorat de sciences de gestion, IAE Poitiers, 1996

SIMON G., Puissance sportive et ordre juridique étatique, L.G.D.J., 1990.

SIMON G., Le sport dans tous ses pouvoirs, Les cahiers de l'université sportive d'été, 1997.

STIGLITZ J.E., Knowledge as a public good, Mimeo, World Bank, 1999.

BENHAMIAS J-L., Projet d'avis sur le sport professionnel et de haut-niveau, présenté au nom de la section cadre de vie, Conseil économique et social, le 15 mai 2002.

Rapport de la Cour des comptes 2003 (publication février 2004) http://www.ccomptes/publications/rapports/rp2003/chapitreXI.pdf 